\title{
n-3 Polyunsaturated Fatty Acids and Their Role in Cancer Chemoprevention
}

\author{
Zhennan Gu ${ }^{1,2,3} \cdot$ Kai Shan ${ }^{1,2} \cdot$ Haiqin Chen ${ }^{1,2}$ - Yong Q. Chen ${ }^{1,2,3}$
}

Published online: 5 July 2015

(C) Springer International Publishing AG 2015

\begin{abstract}
Polyunsaturated fatty acids (PUFAs), including omega-3 (n-3) and omega-6 (n-6) PUFAs, are essential for human health. Recent research shows n-3 PUFAs and their mediators can inhibit inflammation, angiogenesis, and cancer via multiple mechanisms, including reduced release of $n-6$ fatty acid arachidonic acid from cell membranes, inhibition of enzymatic activities, and direct competition with arachidonic acid for enzymatic conversions. In this review, we discuss inflammation-related cancer, anti-inflammatory effects of $n-3$ PUFA lipid mediators, and antineoplastic activities of $n-3$ PUFA in vitro and in vivo and present an update on recent human trials.
\end{abstract}

Keywords n-3 Polyunsaturated fatty acids - Cancer . Inflammation · Chemotherapy $\cdot$ Lipid mediator

\section{Introduction}

The beneficial effects of omega-3 polyunsaturated fatty acids (n-3 PUFAs) on human health have been known

This article is part of the Topical Collection on Cancer Chemoprevention

Yong Q. Chen

yqchen@wakehealth.edu

1 State Key Laboratory of Food Science and Technology, School of Food Science and Technology, Jiangnan University, Wuxi 214122, People's Republic of China

2 The Synergistic Innovation Center for Food Safety and Nutrition, Wuxi 214122, People's Republic of China

3 Department of Cancer Biology, Wake Forest University School of Medicine, Medical Center Blvd., Winston-Salem, NC 27157, USA for a long time. The idea that dietary PUFAs may be beneficial in preventing disease has first been suggested in the 1970s [1-3]. Studies continue to demonstrate the health benefits of n-3 PUFAs, and in particular, preclinical and clinical studies accumulated more data on the effects of n-3 PUFAs on cancer prevention and suppression [4-10].

The mechanisms of the tumor-suppressing activity of n-3 PUFAs are not yet fully understood. Among other important functions, suppression and resolution of inflammation by n-3 PUFAs and their lipid mediators (LMs) generated much interest $[11 \bullet \bullet, 12 \bullet, 13,14]$. Many lines of clinical evidence suggest that inflammation is a key step in cancer initiation and progression [15]. Aspirin, a non-steroidal anti-inflammatory drug (NSAID), has been shown to have a significant protective effect against cancers by inhibiting inflammation-related enzymes, such as cyclooxygenases (COX) and lipoxygenases (LOX) [16-18]. The enzymatic metabolism of $n-6$ fatty acid arachidonic acid (AA) by these same enzymes leads to the formation of pro-inflammatory and pro-tumorigenic LMs, which promote tumor formation and progression [19]. Recent research showed that n-3 PUFAs have potent effects in inhibiting inflammation, angiogenesis, and cancer via multiple mechanisms, including reduced release of $n-6$ fatty acid AA from cell membranes, inhibition of enzymatic activities, and direct competition with AA for enzymatic conversions $[12 \bullet]$.

There are still inconsistencies in the literature about the inhibitory effects of n-3 PUFAs on cancer, some of which may be due to methodological differences or failing to recognize the importance of n-3 PUFA metabolism $[12 \bullet, 20]$. In this review, we will discuss inflammation-related cancer, antiinflammatory effects of n-3 PUFA LMs, and antineoplastic activities of n-3 PUFAs in vitro and in vivo and present an update on recent human trials. 


\section{Tumor and Inflammation}

The concept that chronic inflammation can trigger tumor initiation and formation was proposed as early as 1863 . Virchow observed that inflammatory cells could infiltrate tumors and accumulate there and hypothesized that cancer arises from inflammatory sites [21]. This concept has been supported by many clinical and research data suggesting that cancers are initiated by chronic inflammatory disease, although many of the cellular and molecular mechanisms mediating this cancer initiation are not fully understood [22].

Inflammation is a beneficial response activated to protect the body from injury and pathogenic infection. Even though this response is necessary for enabling an immune reaction, it may also promote neoplastic diseases. During the cyclically self-stimulating process of inflammation, immune cells express and release inflammation-related cytokines and chemokines such as interleukins (ILs), interferon gamma (IFN $\gamma$ ), and tumor necrosis factor alpha (TNF- $\alpha$ ). These mediators then stimulate other immune cells to activate inflammatory processes. Inflammation has both pro- and antitumorigenic activity [23•]. In the protective scenario, immune cells release inflammatory mediators to attract other immune cells to protect the body from infection and injuries, and these actions are resolved once the threats are diminished. However, in the pro-tumor scenario, the process is interrupted and the balanced control of pro-inflammatory and pro-resolution activities is lost. There is a close link between inflammationrelated illness and cancer. In developing countries, probably due to high incidence of infectious disease, up to $23 \%$ of cancers are initiated by pathogens, including Helicobacter pylori (stomach cancer), hepatitis $\mathrm{B} / \mathrm{C}$ viruses (liver cancer), and human papillomaviruses (HPV, cervical cancers) [24]. Similar links can also be established for other inflammation processes, including prostatitis, leading to prostate cancer; bronchitis, leading to lung cancer; and inflammatory bowel diseases, leading to colorectal cancer [23•]. RAS, MYC, and other oncogenes can cause inflammation-related disease as well. Oncogenes activate inflammatory pathways inside the cell and engage and stimulate neighboring inflammatory cells to create a milieu that weakens anticancer immune defenses [24].

How is the inflammation process associated with cancer initiation? Many cancers start from sites of infection and injury, simply as part of the host reaction for protection. Inflammatory leukocytes, including lymphocytes, macrophages, and neutrophils, migrate to the injured site and produce diverse reactive oxygen and nitrogen species to protect the host from infection and injury. Prolonged infections induce chronic inflammation, which frequently leads to tissue injury and regeneration in the surrounding environment by highly reactive oxidative species secreted from inflammatory cells; these in turn impair DNA replication in proliferating epithelium, causing point mutations, deletions, or rearrangements, and subsequently altering the genome [22]. Yamanishi et al. reported that inflammatory oxidative stress induced p53 mutations in exposed skin and inflamed colon and provided mutant cells in these tissues with a growth advantage [25]. Epidemiological data support the notion that inflammation-related oxidative or nitrosative stress plays an important role in prostate cancer formation, which suggests that the ingestion of dietary antioxidants reduces prostate cancer risk [26]. As a result of these inflammatory reactions, the tumor microenvironment contains innate and adaptive immune cells, cancer cells, and surrounding stromal cells. These different kinds of cells interact with each other directly by contact or indirectly by means of cytokine and chemokine production, and regulate tumor growth. The actions of multiple immune mediators and modulators and of different cell types in the tumor milieu dictate the role that inflammation plays, with pro- or antitumor activity [27]. The pro-tumor activities of inflammatory cells include releasing growth and survival factors, stimulating DNA damage, promoting angiogenesis, as well as facilitating tumor cell invasion by masking tumor cell spreading via lymphatics and capillaries, so that they are not detected by the host defense system [22].

Strong evidence for the involvement of inflammation in cancer development stems from observations that long-term use of traditional non-steroidal anti-inflammatory drugs (NSAIDs) is linked to reduced cancer risk. A clinical cancer prevention study cohort with 662,424 participants reported that frequent aspirin use (at least 16 times per month) was associated with a $40 \%$ reduction in colon cancer-related death over a 6-year period [28]. In another study, aspirin treatment after diagnosis of breast cancer was associated with decreased distant recurrence and mortality due to breast cancer. Compared with the control group, the adjusted relative risks (RRs) for 1,2 to 5 , and 6 to 7 days of aspirin use per week were 1.07 $(0.70-1.63,95 \% \mathrm{CI}), 0.29(0.16-0.52,95 \% \mathrm{CI})$, and 0.36 $(0.24-0.54,95 \% \mathrm{CI})$, respectively [29]. Aspirin can inhibit both cyclooxygenase isoforms Cox-1 and Cox-2, though it inhibits Cox-1 in particular [30]. The Cox-1 gene is constitutively expressed in most tissues, while Cox-2, as an immediate-early response gene, is strongly expressed in many human malignancies [31]. Genetically modified mice deficient in either Cox-1 or Cox-2 provided us with a tool to analyze their functions in carcinogenesis. Mutation in the adenomatous polyposis coli $(A p c)$ gene can results in $100 \%$ of mice having intestinal neoplasia. However, when genetic disruption of the $A p c$ gene was performed in Cox-1- or Cox-2deficient mice, intestinal tumorigenesis was reduced by $80 \%$, implying that the inhibition of COX enzymes could be an alternative anticancer approach [32].

Evidence from human studies also supports the idea that COXs and LOXs play an important role in PUFA metabolism and cancer [33-36], as illustrated in Figure 1. The COX 


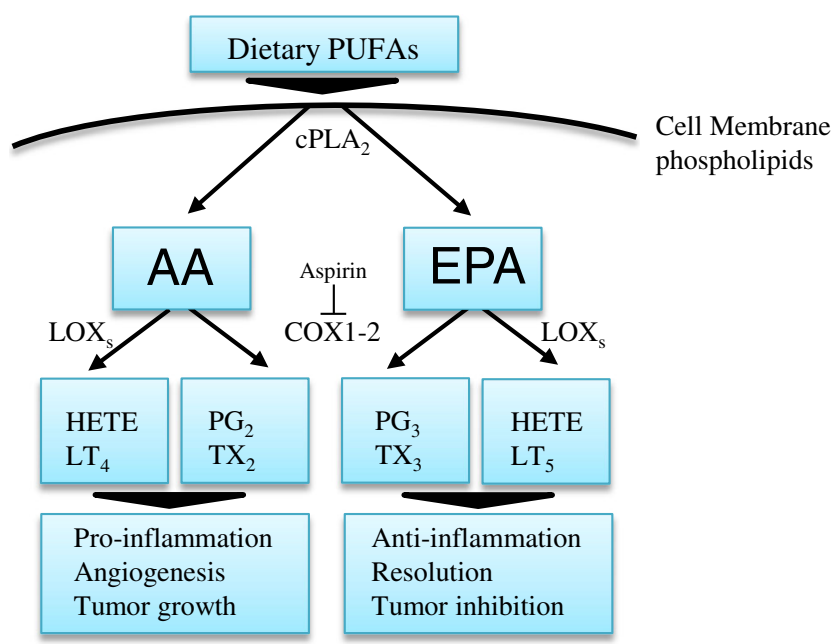

Fig. 1 Conversion of $n-6$ and n-3 PUFAs by eicosanoid pathways. n-6 and n-3 PUFA phospholipids are first released from cell membrane by cytosolic phospholipases A2 (cPLA $)$. These PUFAs are then converted to prostaglandins (PG) and thromboxanes (TX) by cyclooxygenases (Cox1-2) or to leukotrienes (LT) or to hydroxyeicosatetraenoic acids (HETE) by lipoxygenases (LOX). Aspirin can inhibit COX enzyme activity

enzyme produces several major prostanoids, including prostaglandin $\mathrm{D}_{2}$, prostaglandin $\mathrm{E}_{2}$, prostaglandin $\mathrm{F}_{2} \mathrm{a}$, prostaglandin $\mathrm{I}_{2}$, and thromboxane $\mathrm{A}_{2}$. These prostanoids are functionally involved in various biological processes [37]. Among them, $\mathrm{PGE}_{2}$ is highly expressed in different human cancers, including breast, lung, colon, and head and neck cancers. The upregulation of PGE2 is linked to poor disease prognosis. In addition, $\mathrm{PGE}_{2}$ is able to suppress immune response locally to exert its potent pro-tumorigenic activity [23•]. COX-2 is highly activated in inflammation; it has been the focus of intensive study and was suggested as a cancer therapeutic target [37-39]. COX-2derived mediators also play an important role in the early response of the inflammatory reaction and the resolution of inflammation. NSAIDs, such as aspirin, downregulate prostaglandin levels by inhibiting COX enzymes, to exert their antiinflammatory and antitumor activity [23•].

\section{Antineoplastic Activities of n-3 PUFAs}

Total fat consumption and the n- $6 / \mathrm{n}-3$ PUFA ratio (the ratio of n-6 to $n-3$ fatty acids) in the Western diet have increased dramatically since the Industrial Revolution [40]. Diets rich in n6 PUFA induce macrophage infiltration and activate inflammation-related pathway, such as TNF- $\alpha, \mathrm{PGE}_{2}, \mathrm{NF}-\mathrm{\kappa} \beta$, and Wnt signaling, and lead to an increased risk of tumorigenesis [41]. The consumption of high-fat food has been associated with increased incidence of certain types of cancer such as colon, breast, prostate, and pancreatic cancers. n-3 PUFA should not be included in this high-fat category, since diets rich in n-3 PUFA have a protective effect against colon, prostate, and breast cancer in a number of experimental systems [4, 8, 42-47]. Most of the epidemiological studies, including correlational and migrational studies on the association of n-3 PUFAs and cancer, suggest that n-3 PUFAs have a protective and n-6 PUFAs have a promoting effect on cancer development. However, some reviews suggested differently $[48,49]$. Several confounding factors could contribute to the inconsistent results about the effects of n-3 PUFAs on prostate cancer. Population-based studies mostly rely on data from self-reported dietary fat consumption or from assessments based on national dietary habits, and these evaluations can be poorly correlated to real fatty acid composition in patient samples by direct measurements. In addition, the actual amount of n-3 PUFA consumption may be too low to have a protective effect in some cases. Similarly, the ratio of n- 6 to n3 fatty acids may be more important than the absolute amount of n-3 PUFA, as suggested by animal and human studies [8]. Using a phosphatase and tensin homolog (Pten)-null mouse prostate cancer orthotopic model, we demonstrated that when the ratio of $n-6$ to $n-3$ is equal to or less than $5, n-3$ PUFAs were effective in slowing cancer progression [44]. DiNicolantonio et al. evaluated the effects of long-term fish oil consumption on cancer risk and proposed several conclusions in support of their original hypothesis that a diet rich in EPA/DHA reduces the risk of various adenocarcinomas by blocking $\mathrm{PGE}_{2}$ production and activity and does not increase the risk of vascular health like COX-2-specific NSAIDs do [50].

Dietary PUFAs can alter the structure of glycerophospholipids in cell membranes by switching fatty acids. The sn-1 position on the glycerol backbone of glycerophospholipids in mammals is mainly committed to a saturated fatty acid such as stearic acid (SA, 18:0), while the sn-2 position is devoted to an n-6 PUFA, such as AA. Providing animals or cultured cells with n-3 PUFAs can substitute n-6 with n-3 fatty acids at the sn- 2 position of glycerophospholipids. The n-6 to n-3 fatty acid switch can be considered as a dietdriven sn-2 fatty acid moiety change [51-53]. Dietary PUFAs not only change the sn-2 fatty acid moiety but can also influence the fatty acid composition of glycerophospholipids in cell membranes. We have found that approximately $25 \%$ of input fatty acids conjugated with albumin is incorporated into glycerophospholipids in prostate cancer cells within $48 \mathrm{~h}$. This shows that dietary PUFAs can influence the fatty acid composition of glycerophospholipids in cell membranes [51].

A common fate of unsaturated lipids released from the membrane is oxidation. $n-6$ PUFA AA is released from phospholipids by phospholipase $A_{2}$, an enzyme that can be activated by inflammation. The free AA is then processed through a series of enzymatic reaction by several enzymes belonging to the COX and LOX families as well as cytochrome P450, to generate prostaglandins (PGs), thromboxanes, leukotrienes (LTs), hydroxyeicosatetraenoic, and epoxyeicosatrienoic acid, 
respectively. These eicosanoids are potent mediators of inflammation [54-56].

Although the metabolism of n-3 PUFAs is not yet fully understood, studies of n-3 PUFA-derived lipid mediators have been initiated a long time ago. Bang et al. first associated the diet rich in fish of Greenland Eskimos with their lower mortality rate from coronary heart disease and lower prevalence of inflammation-related diseases, such as inflammatory bowel disease, rheumatoid arthritis, psoriasis, asthma, and other autoimmune diseases [57, 58]. With a modern lipidomics approach, Serhan and colleagues discovered and named the EPA-derived resolvins of E series (RvE1 and RvE2), DHA derived resolvins of D series (RvD1 and RvD2) and (neuro-)protectin (PD1) from resolving exudates of mice fed with n-3 PUFAs or treated with aspirin [59]. The E series resolvins are endogenously expressed lipid mediators with anti-inflammatory and proresolving functions [60]. With their anti-inflammatory capacity, RvE1 and RvE2 have shown their protective character in various animal models of disease. For example, RvE1 acted as a protector to resolve inflammation of periodontal disease trigged by bacterial infection [61] and to prevent oxygen-induced retinal angiogenesis [62]. The D series resolvins, another family of endogenously expressed lipid mediators, are also heavily involved in the resolution of inflammation [60, 63].

Not only functioning as precursors to eicosanoids and other metabolites, n-3 PUFAs can also exert their function by directly inhibiting the biosynthesis of $n-6$ series eicosanoids. As mentioned earlier, when n-3 PUFAs are integrated into membrane phospholipids, they take the place of AA at the sn-2 position on the glycerophospholipid backbone and thereby reduce the amount of AA available for cell metabolism. Since n-3 and n6 PUFAs are metabolized by the same enzymes, such as desaturases, elongases, COXs, and LOXs, n-3 PUFAs compete with n-6 PUFAs for these enzymes and inhibit biosynthesis of n6 series eicosanoid [4]. Several groups reported that n-3 PUFAs counter-regulate AA-derived eicosanoids in cells, animals, and humans by inhibiting n-6 PUFA metabolism and antagonizing them on their oxygenation pathways to produce mediators [23•, $61,64]$. DHA can downregulate the formation of AA-derived $\mathrm{PGE}_{2}$. n-3 PUFAs inhibited tumor cell growth and invasion in a xenograft animal model, and the inhibition was associated with decreased levels of both COX-2 and $\mathrm{PGE}_{2}$ [65]. Thus, dietary n3 PUFAs may function as natural COX inhibitors.

Integration of PUFAs into glycerophospholipids is a rapid and efficient process. We reported that about $25 \%$ of input $n-3$ fatty acids in albumin-conjugated form was integrated into cell membranes in 2 days. The majority of these newly integrated PUFAs were in the form of phosphatidylcholine (PC) and phosphatidylserine (PE) [51]. It was also reported that intravenous injection of omega-3 PUFAs triggered a rapid increase of EPA in erythrocytes and of EPA and DHA in plasma PC [66].
The membrane integration of n-3 PUFAs modifies glycerophospholipid structure. Fatty acid at the sn-2 position of the glycerol backbone can be replaced by DHA, and this replacement also changed the species of phospholipid on the membrane. DHA phospholipids inhibited $\mathrm{AKT}^{\mathrm{T} 308}$ but not $\mathrm{AKT}^{\mathrm{S} 473}$ phosphorylation, altered the localization of $\mathrm{PIP}^{3}$ and phospho- $\mathrm{AKT}^{\mathrm{S} 473}$ protein in the cell, decreased the interactions of pPDPK $1{ }^{\mathrm{S} 24} 1-\mathrm{AKT}$ and AKT-BAD, and suppressed the growth of prostate cancer. Knockdown of Bcl-2-associated death promoter (BAD) abolished n-3 PUFA-induced cell apoptosis, and introduction of exogenous BAD restored cancer cell sensitivity to n-3 fatty acid treatment in vitro. Knockout of BAD eliminated the inhibitory effect of n-3 PUFA on the growth of prostate tumor in an animal model. These data suggest that inhibition of prostate cancer growth by $n-3$ PUFAs is modulated in part via the PI3K/AKT/BAD signaling pathway [51].

Several groups have recently reported that unconjugated free fatty acids could activate $G$ protein-coupled receptors (GPCRs), a family of transmembrane proteins, including GPR40, GPR41, GPR43, GPR84, and GPR120. They found that GPR120 can function as a long-chain n-3 PUFA receptor both in vitro and in vivo and hypothesized that reduced GPR120 activity can be an important factor for tissue inflammation, insulin resistance, and obesity [67]. GPR120 receptor expression is upregulated in pro-inflammatory bone marrowderived $\mathrm{CD}_{11 \mathrm{C}^{+}}$macrophages (BMDCs), monocytic macrophage cells, adipose tissue, and mature adipocytes. In GPR120 receptor-positive cells, DHA strongly inhibited lipopolysaccharide-induced phosphorylation of JNK and IKK $\beta$, degradation of IKB protein, secretion of proinflammatory cytokines, and expression of inflammationrelated genes. The inhibitory effects of DHA were completely abolished by knocking down the GPR120 gene, indicating that the anti-inflammatory effects of DHA were specifically modulated through the GPR120 receptor [67].

Toll-like receptors (TLRs), a family of transmembrane glycoprotein receptors, play an important role in the innate immune system. TLR expression is upregulated in the microenvironment of many types of tumors, including breast, prostate, lung, pancreatic, and liver cancer [68]. TLRs activate the production of many inflammation-related cytokines via a signaling cascade. These cytokines then associate with components of the adaptive immune system to destroy intruders [69]. Among the TLR family, TLR4 and TLR9 have been reported to be associated with prostate cancer, while TLR3 can induce human prostate cancer cell apoptosis through a PKC-alphadependent mechanism [70-72]. Huang et al. suggested that saturated fatty acids promoted TLR2 and TLR4 mediated pro-inflammatory activity in a cell-based system, while DHA suppressed such TLA receptor-mediated activity [73].

Syndecan-1 (SDC-1) is another cell membrane protein which can be functionally modulated by $n-3$ PUFAs. SDC-1 
protein functions as an integral membrane protein and participates in recruitment of leukocytes in non-infectious inflammatory diseases, inhibition of inflammation by attenuating heparin sulfate chain-binding pro-inflammatory factors, and remodeling of injured cardiac tissues [74]. Hu and colleagues found that n-3 PUFA-induced prostate cancer cell apoptosis occurred through modulation of SDC-1 expression, followed by downregulation of PDPK1/AKT/BAD phosphorylation [75]. Loss of cell surface expression of SDC-1, as seen in many cancers such as colorectal and skin cancer, triggers metastatic transformation in cancer cells [74]. Our own studies have found that in contrast to normal prostate epithelial cells and androgen-independent PC 3 and DU145 cells, SDC-1 protein expression was downregulated in prostate cancer cell lines and androgen-dependent lymph node carcinoma of the prostate (LNCaP) cells [75]. In a Pten-null mouse prostate cancer model, we have also found that inhibition of prostate cancer growth by dietary n-3 PUFAs was associated with an increased expression of SDC-1 protein $[75,76]$.

Hepatocellular carcinoma (HCC) is primarily a consequence of long-term chronic liver inflammatory diseases of various origins. Weylandt et al. used the fat 1 transgenic mouse model, which endogenously converts n-6 PUFAs to n-3 PUFAs, to analyze the effect of n-3 PUFAs on liver cancer formation in chemical carcinogen-induced tumors in vivo. By comparing the size and number of surface-visible tumors in the liver, they found that increases in n-3 PUFA levels in tissues resulted in a decrease in tumor formation. They also found that inflammation-related markers, such as liver COX-2 gene expression and plasma TNF- $\alpha$ protein, were significantly lower in fat-1 transgenic mice. Their results strongly indicate that high levels of n-3 PUFAs in tissues inhibit liver carcinogenesis, probably through downregulation of the inflammatory response [77]. Lim and colleagues reported that n-3 PUFAs EPA and DHA inhibited HCC development by inhibiting the expression of both COX-2 and $\beta$-catenin. EPA and DHA treatment resulted in a reduction of cell viability in a dose-dependent manner, accompanied with the cleavage of PARP, caspase-3, and caspase-9 in human HCC cell lines, while n-6 PUFA AA had little effect. By downregulating COX-2 and upregulating COX-2 antagonist $15-$ hydroxyprostaglandin dehydrogenase (15-PGDH), DHA suppressed $\mathrm{PGE}_{2}$ signaling and inhibited the growth of inflammation-related HCC in vivo [78].

In a 22-year prospective cohort study with 500 confirmed cases of colon and colorectal cancer, Hall et al. studied the association between $n-3$ PUFAs/marine fish consumption and the risk of colon and colorectal cancer. Marine fish consumption was inversely related to the risk of colorectal cancer. The multivariate RR for people consuming fish more than five times per week compared to those less than once per week was 0.63 (95\% CI, 0.42-0.95). Intake of n-3 fatty acids had very similar results compared to marine fish consumption.
The authors further analyzed the association between fish consumption and the risk of colon and rectal cancers separately. The RRs were 0.62 (95\% CI, 0.38-1.00) for colon cancer and 0.65 (95\% CI, 0.30-1.41) for rectal cancer. These results are consistent with their previous study comparing blood levels of $\mathrm{n}-3$ PUFAs in a case-control study within the same cohort. The findings from this long-term prospective study support the idea that consumption of marine fish and n-3 PUFAs may reduce colorectal and colon cancer risk $[79,80]$.

\section{Combinational Adjuvant Therapy with n-3 PUFAs}

Although many improvements have been made in recent years, traditional cancer chemotherapy alone often does not provide satisfactory long-term clinical results. In most cases, only partial response is achieved. Even with complete remission cases, cancer cells often continue to proliferate and eventually metastasize. Combining agents result in superior response rates and increased disease-free or even overall survival. However, combinational chemotherapy has also been associated with increased treatment complexity and toxicity and, frequently, decreased quality of life (QoL) [81]. n-3 PUFAs, as we discussed above, have potent anticancer effects with multiple targets, including anti-inflammation, inducing cell apoptosis, etc. Thus, n-3 PUFAs are good candidates for chemoprevention or combinational chemotherapy. Most importantly, n-3 fatty acids can serve as a "health promoter" to increase the QoL of cancer patients. For instance, plasma n-3 PUFA levels in cancer patients are up to $50 \%$ lower than in healthy individuals; low $n-3$ fatty acids are related to loss of adipose tissue and skeletal muscle, an indication of poor treatment response and reduced survival of cancer patients, thus suggesting supplementation with n-3 PUFA will be beneficial. Many studies show prevention of muscle loss or gain of body mass with n-3 PUFA supplementation during cancer chemotherapy. n-3 PUFA supplementation may not only improve the cachexia condition for cancer patients but also deliver better response to treatment and reduce side effects associated with cancer chemotherapy [3].

COX-2 inhibitors may be effective chemopreventive compounds against colorectal cancer (CRC), but their therapeutic applications have been snubbed because of severe cardiovascular and gastrointestinal side effects. Fini et al. found that, in a mouse colon cancer model, a diet rich in EPA in the form of free fatty acids significantly suppressed polyp number (by $71.5 \%$ in the small intestine and $78.6 \%$ in the colon, $P<0.0001$ ) and load (by $82.5 \%$ in the small intestine and $93.4 \%$ in the colon, $P<0.0001$ ), with a reduction in COX-2 expression and $\beta$-catenin nuclear translocation [82]. In spite of significant developments in cytotoxic chemotherapy and targeted therapy in the past decade, therapies for colon cancer still remain ineffective $[83,84]$. Thus, n-3 PUFAs used as 
chemoimmunotherapeutic agents in association with cytotoxic chemotherapeutic agent will be a good candidate for colon cancer combinational chemotherapy. In a cell-based experiment, Granci et al. treated colon cancer cells with a combination of n-3 PUFA and cytotoxic chemotherapeutic agent 5fluorouracil and found a significant increase in apoptosisrelated mitochondrial membrane depolarization [85]. However, it is likely that this combination treatment only relied on the capacity of n-3 PUFA to induce cancer cell apoptosis and not on its anti-inflammatory effects, since there were no immune cells to elicit an immune response in their cell-based system.

Polycomb group protein EZH2 is overexpressed in patients with prostate cancer, breast cancer, and other neoplasia. Histological analyses have shown that EZH2 expression increased in normal breast epithelium of patients with a higher risk of developing breast cancer. It has been suggested as a marker for aggressive breast cancer [86]. Dimri and colleagues found that dietary n-3 PUFAs can suppress EZH2 expression in breast cancer cells. Treating breast cancer cells with n-3 PUFAs caused an inhibition of EZH2 expression and a reduction in cancer cell invasion, an oncogenic characteristic that is associated with EZH2. The authors proposed that n-3 PUFAs may exert their anti-oncogenic and chemopreventive effects by suppressing EZH2 gene expression [86]. Patients with breast cancer often develop bone metastasis, which causes severe pain associated with osteolytic lesions and bone fracture. Based on a mouse bone metastasis model of MDAMB-231 human breast cancer cells, Mandal and colleagues reported that a marine fish oil-rich diet inhibits the development of osteolytic lesions in the bone, indicating suppression of cancer cell invasion. The mechanism through which n-3 PUFAs inhibit metastasis of breast cancer cells to the bone still needs to be clarified [87]. Our previous studies showed that DHA supplementation induced SDC-1 expression and trigged human breast cancer cell apoptosis in vitro. By interacting with the SDC-1 ectodomain, DHA inhibited the phosphorylation of MAPK/Erk (MEK)/extracellular signalregulated kinase (Erk) and BAD to induce cell apoptosis. Knocking down SDC-1 expression by siRNA abolished the inhibitory effects of DHA on the phosphorylation of these signaling molecules and diminished cancer cell apoptosis. In the Fat $-1^{\mathrm{T}}$ transgenic mouse model, a genetic model able to convert endogenous n-6 to n-3 PUFAs, SDC-1 levels were higher in Fat $-1^{\mathrm{T}}$ mammary tissue compared with that of wild-type (wt) mice. Phosphorylation of MEK, Erk, and BAD was lower in the Fat- $1^{\mathrm{T}}$ vs. wt tissue. Phosphorylated MEK, Erk, and BAD were markedly higher in mammary gland tissue of Fat- $1^{\mathrm{T}} / \mathrm{SDC}-1^{(--)}$mice compared with those of SDC- $1^{(+/+)}$mice. These results indicate that SDC-1, upregulated by DHA, promotes apoptosis in breast cancer cells through downregulation of MEK/Erk/BAD signaling [88]. All these in vitro and in vivo results demonstrated that $n-3$ PUFAs can downregulate cancer-related cellular proteins and modify cellular signaling to inhibit tumor growth and metastasis. Further studies could be done in these models to see if n-3 PUFAs can be synergistically combined with other chemotherapy or chemoimmunotherapy agents, as a multitargeted approach to treat breast cancer or contain its metastasis.

\section{Hormonal Therapy and Potential Prevention of Bone Loss with n-3 PUFAs}

Steroid hormones have been associated with all stages of breast and prostate cancer development. Hormone deprivation therapy for breast and prostate cancer has helped greatly in preventing disease recurrence or prolonging time to recurrence, but eventually, some tumors begin to resist the treatment and relapse to hormone-independent forms. Progression to resistance to hormone therapy in both cancers causes tens of thousands of patient deaths each year [89, 90]. Cancer hormone deprivation therapy is often followed with severe bone loss and high risk of bone fracture. Ovarian ablation therapy in premenopausal women with breast cancer is associated with a high incidence of bone loss, as high as $13 \%$ within 12 months of treatment [91]. Likewise, androgen deprivation therapy triggered significant loss of bone density, about 6-7\% within the first 18 months of treatment [92].

Several in vitro and in vivo experiments suggest that $n-3$ PUFAs may be effective in slowing down androgenindependent (AI) prostate cancer growth. Mice with deletion of the c-Jun $\mathrm{NH}_{2}$-terminal kinase (JNK) or phosphatase and tensin homolog (Pten) gene can develop invasive AI prostate cancer [93]. Knock-out of Pten expression increased the expression of androgen receptor (AR) and prostate cancer cells became more resistant to castration treatment [46]. The increased expression of both androgen receptor mRNA and protein is necessary and sufficient to transform prostate cancer from hormone-sensitive to hormone-insensitive [94]. In a Pten-null prostate cancer model, we found that n-3 PUFAs decrease the development of castration-resistant tumors as compared with $\mathrm{n}$ 6 PUFA. n-3 PUFAs downregulated AR protein levels in both cytosolic and nuclear fractions of tumor cells but had little effect on AR mRNA levels. n-3 PUFA-induced degradation of AR protein could be blocked by proteasome inhibitor MG132. Reducing the expression of AR significantly reduced prostate cancer cell proliferation. These results indicate that n-3 PUFAs prevent or delay androgen-independent prostate cancer development in part by degrading AR protein in a proteasomedependent manner [46]. In a mouse CWR22 androgenindependent prostate cancer xenograft model, McEntee et al. found that there was a highly significant positive correlation between the ratio of apoptosis to mitosis and total n-3 PUFAs in tumors and with the n-3/n-6 PUFA ratio, and an inverse correlation with tissue AA and total n-6 PUFA content [95]. In a cell-based study, Friedriches et al. found that EPA and 
DHA were able to slow down the growth of LNCaP cells, an androgen-dependent prostate cell line, while AA increased androgen-independent prostate cancer cell growth. Their results established a possible correlation between decreased expression of the androgen receptor and suppression of the Akt/mTOR signaling pathway [96]. In a Pten-null mouse model, where AKT is constitutively active, we also found that n-3 PUFAs can inhibit prostate cancer growth by inhibiting the PI3K/AKT survival pathway [51]. Thus, supplementing dietary n-3 PUFAs in combination with androgen ablation therapy maybe more effective in preventing or inhibiting the development of androgen-independent prostate cancer in patients compared with androgen ablation therapy alone.

Similarly, in vitro and in vivo experiments also suggest the potential use of n-3 PUFA to prevent the development of estrogen-independent breast cancer and its metastasis. Regulation of hormonal steroidogenesis involves the metabolism of AA via the 5-lipoxygenase pathway. Cooke et al. reported that AA metabolites contribute to steroidogenic acute regulation by engaging, at least in part, the autocrine- or paracrineactivated eicosanoid receptor, OXE-R. DHA treatment partially but significantly decreased progesterone production in OXE-R-expressing cells. These results suggest that DHA can antagonize AA-regulated steroidogenesis through the 5lipoxygenase pathway [97]. Traditionally, $17 \beta$-estradiol (E2) has been considered as an estrogen receptor- $\alpha(\mathrm{ER} \alpha)$ activator to promote breast cancer growth. Although inhibition of ER $\alpha$ is a successful approach for patients with ER $\alpha$-positive tumors, some patients become resistant to anti-estrogen therapy after an initial response. Subsequent studies revealed that E2 could exert its activity through $\mathrm{G}$ protein-coupled estrogen receptor (GPER). Marjon et al. provided the first in vivo evidence that GPER played a critical role in breast tumor growth and metastasis [98]. Cao and colleagues showed that EPA and DHA could switch the effects of estrogen from pro-survival and proliferative to pro-apoptotic in human breast cancer cell lines. EPA and DHA promoted such pro-apoptotic action of estrogen by sensitizing the GPER1-cAMP-PKA pathway and blunting the response of EGFR, Erk1/2, and AKT signaling [99]. Based on the known functional interaction between the estrogen and PPAR $\gamma$ receptors, Manni et al. tested the hypothesis that the combination of estrogen receptor antagonist tamoxifen with n-3 PUFAs would have a better antitumor effect than either agent alone. In a chemical carcinogen-induced mammary carcinogenesis mouse model, they demonstrated that the combination of tamoxifen and n-3 PUFAs could inhibit tumor development and proliferation to a greater extent than the individual interventions [100]. These results provide a positive indication of better efficacy of escalating n-3 PUFA biologic effects and the pro-apoptotic signaling of estrogen in breast cancer cells and also provide us with a new vision for the potential application of n-3 PUFAs combined with hormone therapy for breast cancer.
The sex hormones are particularly important for adults to maintain healthy bones. Loss of hormone production or reduced levels may lead to bone loss. It has been reported that hormonal therapy for women with breast cancer can reduce bone density at a significant rate, at least double that of women during early menopause [101]. For men receiving hormone therapy during prostate cancer treatment, a significant drop of hip bone mineral density occurs frequently, up to $9.6 \%$ bone loss in the first year post treatment [102]. A recent study reported that lower risk of hip fracture was associated with higher red blood cell AA (hazard ratio (HR) 0.44), EPA (HR 0.46), and total n-3 PUFAs (HR 0.55). More importantly, the incidence of hip fractures nearly doubled with the highest red blood cell n-6/n-3 ratio (HR 1.96) [103]. A positive correlation between n-3 PUFA levels in erythrocytes and bone mass was also reported in postmenopausal Korean women with osteoporosis [104]. In Fat-1 transgenic mice with high endogenous n-3 PUFA levels, there was no significant bone loss after ovariectomy, while there was substantial bone loss after ovariectomy in wild-type mice [105]. Endogenously produced n-3 PUFAs can attenuate ovariectomy-induced bone loss, probably by reducing adipogenesis of bone marrow and osteoclastogenesis [106, 107].

Among several potential mechanism whereby n-3 PUFAs may affect the bone, anti-inflammation may be critical. Cytokines are key regulators controlling the ratio of osteoprotegerin (OPG) and receptor activator of NFkB ligand (RANKL) in bones. RANKL is expressed in osteoblasts, while RANKL receptor RANK is expressed on osteoclasts. RANKL binds to RANK on osteoclasts to activate the receptor and stimulate osteoclast formation and to suppress osteoclast apoptosis. OPG is a glycoprotein expressed by and secreted from osteoblasts. OPG can function as an antagonist blocking RANKL and preventing it from activating RANK. The ratio of OPG/ RANKL is important for bone health, with a higher ratio indicating a high bone density, or less bone resorption [108]. Sub-physiologic concentrations of testosterone increase inflammation and bone loss in male rats by decreasing the ratio of OPG/RANKL. n-3 PUFA mediator resolvin D2 (RvD2) can partially reverse this impact and ameliorate low testosterone-derived inflammatory response [109]. In transgenic mice overexpressing the pro-resolving RvE1 receptor on leukocytes, local RvE1 application during uniform craniotomy in the parietal bone significantly enhanced the expression of OPG and subsequently enhanced the regeneration of the bone defect [110]. n-3 PUFAs have been reported to downregulate the production of $n-6$ FA-derived eicosanoid $\mathrm{PGE}_{2}$. High $\mathrm{PGE}_{2}$ decreases OPG production and increases RANKL expression [111]. Thus, DHA can increase the OPG/RANKL ratio by decreasing $\mathrm{AA}-$ mediated $\mathrm{PGE}_{2}$. These data suggest that the optimal balance of n-3 and n-6 PUFAs is important in order to maintain a less inflammatory cytokine environment favorable to bone health. 


\section{Clinical Trials Using n-3 PUFAs for Cancer Prevention and Treatment}

Based on the epidemiological data and the demonstrated multitargeted effects of n-3 PUFAs on cancer in cell culture and animal models, many clinical intervention trials were proposed and developed to validate the effectiveness of fish oil or n-3 PUFAs in cancer prevention and treatment or to provide nutritional support for cancer patient who suffered weight loss, fatigue, and other inflammation-related illness [4]. Here we will update our previous review with recent clinical trials.

Several clinical trials address the potential applications of n-3 PUFAs in treating patients with cancer-associated weight loss (cachexia). Studies show that an inflammatory reaction to a local tumor can also trigger a cascade of systemic inflammation that eventually lead to development of anorexia and catabolic processes, such as muscle proteolysis and lipolysis, the early stage of fatigue and cachexia [112]. Several clinical trials have been reported using EPA or marine fish oil (EPA + DHA) in purified form or in the form of oral supplements in an effort to prevent or reduce weight and muscle loss in cancer patients. The studies show that EPA supplementation with a dosage of more than $2 \mathrm{~g}$ per day can reduce, even stabilize, weight loss and lean tissue wasting in patients with advanced stages of cancer [112-115].

However, recent data from a double-blind placebo-controlled randomized clinical trial suggested that EPA supplementation alone is not effective in treating cancer cachexia [116]. By comparing the effects of an EPA supplement, megestrol acetate (MA), and combination treatment, another trial found that the EPA supplement, either alone or in combination with MA, did not improve weight or appetite better than MA alone. EPA was comparable to MA with respect to appetite gain, quality of life, and survival rate. Combination therapy did not have additional benefits relative to MA alone [117]. In this trial, the dosage of EPA supplement was $1.09 \mathrm{~g} /$ day, lower than the effective dosage ( $>2.0 \mathrm{~g} /$ day) suggested by another trial [116]. Successful management of cachexia may require a multimodal approach with nutritional supplementation and pharmacological treatment. A recent randomized phase III clinical trial suggested that combination therapy with MA, EPA supplementation, thalidomide, and L-carnitine was significantly more effective in improving lean body mass and appetite than single agents [118]. Combination pharmacological therapy with nutritional supplementation of $n-3$ PUFAs may be the appropriate approach to cancer cachexia management.

Nutritional supplements of n-3 PUFAs as immunomodulators have also been studied in several clinical trials for their ability to prevent inflammation and infection. Administration of n-3 PUFAs pre- or post-surgery of major abdominal cancer was shown to decrease inflammatory cytokine levels [4]. In a randomized intervention trial, patients with elective colorectal cancer surgery were given oral nutritional supplements enriched with n-3 PUFAs at dosage of $2.0 \mathrm{~g}$ of EPA and $1.0 \mathrm{~g}$ of DHA per day for 7 days before surgery. $n-3$ PUFAs increased the levels of anti-inflammatory modulators leukotriene B5 (LTB5) and 5-hydroxyeicosapentaenoic acid (5-HEPE) and reduced the production of leukotriene B4 (LTB4), a pro-inflammatory modulator. The trial concluded that orally administrated n-3 PUFAs can exert antiinflammatory effects in surgical patients, while having no effect on reducing the risk of postoperative complications [119]. A clinical phase II trial to improve outcome of chemotherapy in metastatic breast cancer by DHA was based on the finding that compared to normal cells, tumor cells can be more easily sensitized to chemotherapy when membrane lipids contain high level of DHA, because of differential antioxidant defense level between normal and tumor cells. After administration of DHA at a dosage of $1.8 \mathrm{~g}$ per day to anthracycline-based chemotherapy patients with breast cancer and visceral metastases, DHA level was $5.1 \%$ of total fatty acids on average (range 2.5-8.3\%) while baseline DHA level was $2.6 \%$ (1.65). The trial results showed that time to progression (TTP) was 8.7 months in the high $(\mathrm{H})$ DHA group (plasma DHA $>2.5 \%$ ) vs. 3.5 months in the low (L) DHA group (plasma DHA $<2.5 \%$ ). The overall survival was significantly greater in the H-DHA group with a median survival time of 34 months vs. 18 months in the L-DHA group $(P=0.007)$. The study concluded that DHA had no adverse side effects, unlike chemotherapy, and improved the outcome of treatment; at high plasma level, DHA has a potential to specifically chemosensitize tumors for better therapeutic effects [120].

Some studies suggested that COX inhibitors, primarily blocking AA metabolism, are effective in the prevention of prostate and colon cancer. However, the severe cardiovascular side effects of COX-2 inhibitors have threatened the clinical application of these inhibitors [4]. To examine the beneficial effects of modulating dietary fat content and the n- $6 / n-3$ PUFA ratio in prostate cancer patients on the insulin-like growth factor/insulin-like growth factor-binding protein (IGF/IGFBP) system and the COX-2/PGE 2 pathways, Aronson et al. initiated a phase II prospective randomized trial focused on lowfat diet supplemented with fish oil in patients undergoing radical prostatectomy. In the low-fat fish oil vs. Western diet group, $n-6 / n-3$ ratios were reduced in benign and malignant prostate tissue, cell proliferation was reduced as determined by a reduced proliferation index (Ki67) both in vivo and in vitro, and there was no significant difference in mean $\mathrm{PGE}_{2}$ levels, COX-2, apoptosis (TUNEL), or angiogenesis (CD-31) immunostaining [121].

\section{Conclusion}

High n-6 to n-3 PUFA ratio in the Western diet may be associated with high cancer incidence and mortality in the Western 
world. Bringing back a healthier, lower n-6 to n-3 PUFA ratio is an attractive approach for cancer prevention. Many of the anticancer effects of n-3 PUFAs observed in animal models and cell-based systems failed to translate into human trials or to be confirmed in some observational human studies. However, one potential factor at the origin of this discrepancy is that those studies did not take into account the importance of the $n-6 / n-3$ PUFA ratio. Therefore, establishing an n-6/n-3 PUFA index for individual patients will become more important in the future n-3 PUFA cancer intervention or chemoprevention trials in order to provide the optimal dose of $n-3$ PUFAs leading to the proper n-6/n-3 PUFA ratio.

\begin{abstract}
Acknowledgments This study was supported in part by the National Science Foundation of China (NSFC) $(21276108,31471128,31400038)$, the Program for New Century Excellent Talents (NCET-13-0831), the Program for Changjiang Scholars and Innovative Research Team in University (IRT1249), the Fundamental Research Funds for the Central Universities (JUSRP51320B), and National Institutes of Health grants (R01CA107668, R01CA163273).
\end{abstract}

\section{Compliance with Ethics Guidelines}

Conflict of Interest Zhennan Gu, Kai Shan, Haiqin Chen, and Yong Q. Chen declare that they have no conflict of interest.

Human and Animal Rights and Informed Consent This article does not contain any studies with human or animal subjects performed by any of the authors.

\section{References}

Papers of particular interest, published recently, have been highlighted as:

- Of importance

•- Of major importance

1. Bang HO, Dyerberg J, Nielsen AB. Plasma lipid and lipoprotein pattern in Greenlandic West-coast Eskimos. Lancet. 1971;1:11435.

2. Dyerberg J, Bang HO, Hjorne N. Fatty acid composition of the plasma lipids in Greenland Eskimos. Am J Clin Nutr. 1975;28: 958-66.

3. Murphy RA, Mourtzakis M, Mazurak VC. n-3 polyunsaturated fatty acids: the potential role for supplementation in cancer. Curr Opin Clin Nutr Metab Care. 2012;15:246-51.

4. Berquin IM, Edwards IJ, Chen YQ. Multi-targeted therapy of cancer by omega-3 fatty acids. Cancer Lett. 2008;269:363-77.

5. MacLennan M, Ma DW. Role of dietary fatty acids in mammary gland development and breast cancer. Breast Cancer Res. 2010;12:211.

6. Gerber M. Omega-3 fatty acids and cancers: a systematic update review of epidemiological studies. Br J Nutr. 2012;107 Suppl 2: S228-39.

7. Sawada N, Inoue M, Iwasaki M, Sasazuki S, Shimazu T, et al. Consumption of n-3 fatty acids and fish reduces risk of hepatocellular carcinoma. Gastroenterology. 2012;142:1468-75.
8. Gu Z, Suburu J, Chen H, Chen YQ. Mechanisms of omega-3 polyunsaturated fatty acids in prostate cancer prevention. Biomed Res Int. 2013;2013:824563.

9. Aronson WJ, Glaspy JA, Reddy ST, Reese D, Heber D, et al. Modulation of omega-3/omega-6 polyunsaturated ratios with dietary fish oils in men with prostate cancer. Urology. 2001;58:2838.

10. Norrish AE, Skeaff CM, Arribas GL, Sharpe SJ, Jackson RT. Prostate cancer risk and consumption of fish oils: a dietary biomarker-based case-control study. Br J Cancer. 1999;81:123842 .

11.• Janakiram NB, Mohammed A, Rao CV. Role of lipoxins, resolvins, and other bioactive lipids in colon and pancreatic cancer. Cancer Metastasis Rev. 2011;30:507-23. The paper is a detailed review about lipid mediators and their interactions with immune cells and cytokines in preventing tumor cell proliferation and progression.

12. Wang W, Zhu J, Lyu F, Panigrahy D, Ferrara KW, et al. omega-3 Polyunsaturated fatty acids-derived lipid metabolites on angiogenesis, inflammation and cancer. Prostaglandins Other Lipid Mediat. 2014. The reivew provided a detailed discussion of the biology of cyclooxygenase, lipoxygenase and cytochrome $\mathbf{P 4 5 0}$ enzymes-derived $\omega$-3-series lipid metabolites on angiogenesis, inflammation and cancer.

13. Recchiuti A, Serhan CN. Pro-resolving lipid mediators (SPMs) and their actions in regulating miRNA in novel resolution circuits in inflammation. Front Immunol. 2012;3:298.

14. Fradet V, Cheng I, Casey G, Witte JS. Dietary omega-3 fatty acids, cyclooxygenase-2 genetic variation, and aggressive prostate cancer risk. Clin Cancer Res. 2009;15:2559-66.

15. Landskron G, De la Fuente M, Thuwajit P, Thuwajit C, Hermoso MA. Chronic inflammation and cytokines in the tumor microenvironment. J Immunol Res. 2014;2014:149185.

16. Rothwell PM, Fowkes FG, Belch JF, Ogawa H, Warlow CP, et al. Effect of daily aspirin on long-term risk of death due to cancer: analysis of individual patient data from randomised trials. Lancet. 2011;377:31-41.

17. Chan AT, Ogino S, Fuchs CS. Aspirin use and survival after diagnosis of colorectal cancer. JAMA. 2009;302:649-58.

18. Claria J, Lee MH, Serhan CN. Aspirin-triggered lipoxins (15-epiLX) are generated by the human lung adenocarcinoma cell line (A549)-neutrophil interactions and are potent inhibitors of cell proliferation. Mol Med. 1996;2:583-96.

19. Wang D, Dubois RN. Eicosanoids and cancer. Nat Rev Cancer. 2010;10:181-93.

20. Suburu J, Lim K, Calviello G, Chen YQ. RE: serum phospholipid fatty acids and prostate cancer risk in the SELECT trial. J Natl Cancer Inst. 2014;106:dju023.

21. Balkwill F, Mantovani A. Inflammation and cancer: back to Virchow? Lancet. 2001;357:539-45.

22. Coussens LM, Werb Z. Inflammation and cancer. Nature. 2002;420:860-7.

23. Greene ER, Huang S, Serhan CN, Panigrahy D. Regulation of inflammation in cancer by eicosanoids. Prostaglandins Other Lipid Mediat. 2011;96:27-36. The paper reviewed the actions of eicosanoids on inflammation in the context of tumorigenesis.

24. Trinchieri G. Innate inflammation and cancer: is it time for cancer prevention? F1000 Med Rep. 2011;3:11.

25. Yamanishi Y, Boyle DL, Rosengren S, Green DR, Zvaifler NJ, et al. Regional analysis of p53 mutations in rheumatoid arthritis synovium. Proc Natl Acad Sci U S A. 2002;99:10025-30.

26. Neill MG, Fleshner NE. An update on chemoprevention strategies in prostate cancer for 2006. Curr Opin Urol. 2006;16:132-7.

27. Grivennikov SI, Greten FR, Karin M. Immunity, inflammation, and cancer. Cell. 2010;140:883-99. 
28. Thun MJ, Namboodiri MM, Heath Jr CW. Aspirin use and reduced risk of fatal colon cancer. N Engl J Med. 1991;325:1593-6.

29. Holmes MD, Chen WY, Li L, Hertzmark E, Spiegelman D, et al. Aspirin intake and survival after breast cancer. J Clin Oncol. 2010;28:1467-72.

30. Simmons DL, Botting RM, Hla T. Cyclooxygenase isozymes: the biology of prostaglandin synthesis and inhibition. Pharmacol Rev. 2004;56:387-437.

31. Dubois RN, Abramson SB, Crofford L, Gupta RA, Simon LS, et al. Cyclooxygenase in biology and disease. FASEB J. 1998;12:1063-73.

32. Chulada PC, Thompson MB, Mahler JF, Doyle CM, Gaul BW, et al. Genetic disruption of Ptgs-1, as well as Ptgs-2, reduces intestinal tumorigenesis in Min mice. Cancer Res. 2000;60:4705-8.

33. Pidgeon GP, Lysaght J, Krishnamoorthy S, Reynolds JV, O'Byrne $\mathrm{K}$, et al. Lipoxygenase metabolism: roles in tumor progression and survival. Cancer Metastasis Rev. 2007;26:503-24.

34. Ye YN, Wu WK, Shin VY, Bruce IC, Wong BC, et al. Dual inhibition of 5-LOX and COX-2 suppresses colon cancer formation promoted by cigarette smoke. Carcinogenesis. 2005;26:82734.

35. Sharma BK, Pilania P, Singh P. Modeling of cyclooxygenase-2 and 5-lipooxygenase inhibitory activity of apoptosis-inducing agents potentially useful in prostate cancer chemotherapy: derivatives of diarylpyrazole. J Enzym Inhib Med Chem. 2009;24:60715.

36. Sobolewski C, Cerella C, Dicato M, Ghibelli L, Diederich M. The role of cyclooxygenase- 2 in cell proliferation and cell death in human malignancies. Int J Cell Biol. 2010;2010:215158.

37. Wang MT, Honn KV, Nie D. Cyclooxygenases, prostanoids, and tumor progression. Cancer Metastasis Rev. 2007;26:525-34.

38. Menter DG, Schilsky RL, DuBois RN. Cyclooxygenase-2 and cancer treatment: understanding the risk should be worth the reward. Clin Cancer Res. 2010;16:1384-90.

39. Reese AC, Fradet V, Witte JS. Omega-3 fatty acids, genetic variants in COX-2 and prostate cancer. J Nutrigenet Nutrigenomics. 2009;2:149-58.

40. Simopoulos AP. The importance of the ratio of omega-6/omega-3 essential fatty acids. Biomed Pharmacother. 2002;56:365-79.

41. Kim IW, Myung SJ, Do MY, Ryu YM, Kim MJ, et al. Westernstyle diets induce macrophage infiltration and contribute to colitisassociated carcinogenesis. J Gastroenterol Hepatol. 2010;25: 1785-94.

42. Calviello G, Resci F, Serini S, Piccioni E, Toesca A, et al. Docosahexaenoic acid induces proteasome-dependent degradation of beta-catenin, down-regulation of survivin and apoptosis in human colorectal cancer cells not expressing COX-2. Carcinogenesis. 2007;28:1202-9.

43. Sun H, Berquin IM, Owens RT, O'Flaherty JT, Edwards IJ. Peroxisome proliferator-activated receptor gamma-mediated upregulation of syndecan- 1 by $n-3$ fatty acids promotes apoptosis of human breast cancer cells. Cancer Res. 2008;68:2912-9.

44. Berquin IM, Min $\mathrm{Y}, \mathrm{Wu} \mathrm{R}, \mathrm{Wu} \mathrm{J}$, Perry $\mathrm{D}$, et al. Modulation of prostate cancer genetic risk by omega- 3 and omega- 6 fatty acids. J Clin Invest. 2007;117:1866-75.

45. Chen YQ, Edwards IJ, Kridel SJ, Thornburg T, Berquin IM. Dietary fat-gene interactions in cancer. Cancer Metastasis Rev. 2007;26:535-51.

46. Wang S, Wu J, Suburu J, Gu Z, Cai J, et al. Effect of dietary polyunsaturated fatty acids on castration-resistant Pten-null prostate cancer. Carcinogenesis. 2012;33:404-12.

47. Carroll KK. Dietary fat and cancer: specific action or caloric effect? J Nutr. 1986;116:1130-2.

48. Terry PD, Rohan TE, Wolk A. Intakes of fish and marine fatty acids and the risks of cancers of the breast and prostate and of other hormone-related cancers: a review of the epidemiologic evidence. Am J Clin Nutr. 2003;77:532-43.

49. MacLean $\mathrm{CH}$, Newberry SJ, Mojica WA, Khanna P, Issa AM, et al. Effects of omega-3 fatty acids on cancer risk: a systematic review. JAMA. 2006;295:403-15.

50. DiNicolantonio JJ, McCarty MF, Chatterjee S, Lavie CJ, O'Keefe $\mathrm{JH}$. A higher dietary ratio of long-chain omega-3 to total omega-6 fatty acids for prevention of COX-2-dependent adenocarcinomas. Nutr Cancer. 2014;1-6.

51. Gu Z, Wu J, Wang S, Suburu J, Chen H, et al. Polyunsaturated fatty acids affect the localization and signaling of PIP3/AKT in prostate cancer cells. Carcinogenesis. 2013;34:1968-75.

52. Chabot MC, Schmitt JD, Bullock BC, Wykle RL. Reacylation of platelet activating factor with eicosapentaenoic acid in fish-oilenriched monkey neutrophils. Biochim Biophys Acta. 1987;922: 214-20.

53. Picq M, Chen P, Perez M, Michaud M, Vericel E, et al. DHA metabolism: targeting the brain and lipoxygenation. Mol Neurobiol. 2010;42:48-51.

54. Lewis RA, Austen KF, Soberman RJ. Leukotrienes and other products of the 5-lipoxygenase pathway. Biochemistry and relation to pathobiology in human diseases. N Engl J Med. 1990;323: 645-55.

55. Tilley SL, Coffman TM, Koller BH. Mixed messages: modulation of inflammation and immune responses by prostaglandins and thromboxanes. J Clin Invest. 2001;108:15-23.

56. Kroetz DL, Zeldin DC. Cytochrome P450 pathways of arachidonic acid metabolism. Curr Opin Lipidol. 2002;13:273-83.

57. Bang HO, Dyerberg J, Hjoorne N. The composition of food consumed by Greenland Eskimos. Acta Med Scand. 1976;200:69-73.

58. Dyerberg J, Bang HO, Stoffersen E, Moncada S, Vane JR. Eicosapentaenoic acid and prevention of thrombosis and atherosclerosis? Lancet. 1978;2:117-9.

59. Serhan CN, Clish CB, Brannon J, Colgan SP, Chiang N, et al. Novel functional sets of lipid-derived mediators with antiinflammatory actions generated from omega-3 fatty acids via cyclooxygenase 2-nonsteroidal antiinflammatory drugs and transcellular processing. J Exp Med. 2000;192:1197-204.

60. Serhan CN. Novel lipid mediators and resolution mechanisms in acute inflammation: to resolve or not? Am J Pathol. 2010;177: 1576-91.

61. Gleissman H, Johnsen JI, Kogner P. Omega-3 fatty acids in cancer, the protectors of good and the killers of evil? Exp Cell Res. 2010;316:1365-73.

62. Connor KM, SanGiovanni JP, Lofqvist C, Aderman CM, Chen J, et al. Increased dietary intake of omega-3-polyunsaturated fatty acids reduces pathological retinal angiogenesis. Nat Med. 2007;13:868-73.

63. Serhan CN, Chiang N, Van Dyke TE. Resolving inflammation: dual anti-inflammatory and pro-resolution lipid mediators. Nat Rev Immunol. 2008;8:349-61.

64. Edwards IJ, O'Flaherty JT. Omega-3 fatty acids and PPARgamma in cancer. PPAR Res. 2008;2008:358052.

65. Kobayashi N, Barnard RJ, Henning SM, Elashoff D, Reddy ST, et al. Effect of altering dietary omega-6/omega-3 fatty acid ratios on prostate cancer membrane composition, cyclooxygenase-2, and prostaglandin E2. Clin Cancer Res. 2006;12:4662-70.

66. Al-Taan O, Stephenson JA, Spencer L, Pollard C, West AL, et al. Changes in plasma and erythrocyte omega- 6 and omega- 3 fatty acids in response to intravenous supply of omega- 3 fatty acids in patients with hepatic colorectal metastases. Lipids Health Dis. 2013;12:64.

67. Oh DY, Talukdar S, Bae EJ, Imamura T, Morinaga H, et al. GPR120 is an omega-3 fatty acid receptor mediating potent antiinflammatory and insulin-sensitizing effects. Cell. 2010;142:68798. 
68. Ridnour LA, Cheng RY, Switzer CH, Heinecke JL, Ambs S, et al. Molecular pathways: toll-like receptors in the tumor microenvironment-poor prognosis or new therapeutic opportunity. Clin Cancer Res. 2013;19:1340-6.

69. Krishnan J, Lee G, Choi S. Drugs targeting toll-like receptors. Arch Pharm Res. 2009;32:1485-502.

70. Paone A, Starace D, Galli R, Padula F, De Cesaris P, et al. Toll-like receptor 3 triggers apoptosis of human prostate cancer cells through a PKC-alpha-dependent mechanism. Carcinogenesis. 2008;29:1334-42.

71. Zheng SL, Augustsson-Balter K, Chang B, Hedelin M, Li L, et al. Sequence variants of toll-like receptor 4 are associated with prostate cancer risk: results from the cancer prostate in Sweden study. Cancer Res. 2004;64:2918-22.

72. Vaisanen MR, Vaisanen T, Jukkola-Vuorinen A, Vuopala KS, Desmond R, et al. Expression of toll-like receptor-9 is increased in poorly differentiated prostate tumors. Prostate. 2010;70:81724.

73. Wang Z, Liu D, Wang F, Liu S, Zhao S, et al. Saturated fatty acids activate microglia via Toll-like receptor 4/NF-kappaB signalling. Br J Nutr. 2012;107:229-41.

74. Teng YH, Aquino RS, Park PW. Molecular functions of syndecan1 in disease. Matrix Biol. 2012;31:3-16.

75. Hu Y, Sun H, Owens RT, Gu Z, Wu J, et al. Syndecan-1-dependent suppression of PDK1/Akt/bad signaling by docosahexaenoic acid induces apoptosis in prostate cancer. Neoplasia. 2010;12:826-36.

76. Edwards IJ, Sun H, Hu Y, Berquin IM, O'Flaherty JT, et al. In vivo and in vitro regulation of syndecan 1 in prostate cells by n-3 polyunsaturated fatty acids. J Biol Chem. 2008;283:18441-9.

77. Weylandt KH, Krause LF, Gomolka B, Chiu CY, Bilal S, et al. Suppressed liver tumorigenesis in fat-1 mice with elevated omega3 fatty acids is associated with increased omega-3 derived lipid mediators and reduced TNF-alpha. Carcinogenesis. 2011;32:897903.

78. Lim K, Han C, Dai Y, Shen M, Wu T. Omega-3 polyunsaturated fatty acids inhibit hepatocellular carcinoma cell growth through blocking beta-catenin and cyclooxygenase-2. Mol Cancer Ther. 2009;8:3046-55.

79. Hall MN, Chavarro JE, Lee IM, Willett WC, Ma J. A 22-year prospective study of fish, n-3 fatty acid intake, and colorectal cancer risk in men. Cancer Epidemiol Biomarkers Prev. 2008; 17:1136-43.

80. Hall MN, Campos H, Li H, Sesso HD, Stampfer MJ, et al. Blood levels of long-chain polyunsaturated fatty acids, aspirin, and the risk of colorectal cancer. Cancer Epidemiol Biomarkers Prev. 2007;16:314-21.

81. Huober J, Thurlimann B. The role of combination chemotherapy in the treatment of patients with metastatic breast cancer. Breast Care (Basel). 2009;4:367-72.

82. Fini L, Piazzi G, Ceccarelli C, Daoud Y, Belluzzi A, et al. Highly purified eicosapentaenoic acid as free fatty acids strongly suppresses polyps in $\mathrm{Apc}(\mathrm{Min} /+)$ mice. Clin Cancer Res. 2010;16: 5703-11.

83. Kohne CH, Cunningham D, Di Costanzo F, Glimelius B, Blijham $\mathrm{G}$, et al. Clinical determinants of survival in patients with 5fluorouracil-based treatment for metastatic colorectal cancer: results of a multivariate analysis of 3825 patients. Ann Oncol. 2002;13:308-17.

84. Bloemendaal AL, Verwaal VJ, van Ruth S, Boot H, Zoetmulder FA. Conventional surgery and systemic chemotherapy for peritoneal carcinomatosis of colorectal origin: a prospective study. Eur J Surg Oncol. 2005;31:1145-51.

85. Granci V, Cai F, Lecumberri E, Clerc A, Dupertuis YM, et al. Colon cancer cell chemosensitisation by fish oil emulsion involves apoptotic mitochondria pathway. Br J Nutr. 2013;109:1188-95.
86. Dimri M, Bommi PV, Sahasrabuddhe AA, Khandekar JD, Dimri GP. Dietary omega-3 polyunsaturated fatty acids suppress expression of EZH2 in breast cancer cells. Carcinogenesis. 2010;31: 489-95.

87. Mandal CC, Ghosh-Choudhury T, Yoneda T, Choudhury GG, Ghosh-Choudhury N. Fish oil prevents breast cancer cell metastasis to bone. Biochem Biophys Res Commun. 2010;402:602-7.

88. Sun $\mathrm{H}, \mathrm{Hu} \mathrm{Y}, \mathrm{Gu} \mathrm{Z}$, Owens RT, Chen YQ, et al. Omega-3 fatty acids induce apoptosis in human breast cancer cells and mouse mammary tissue through syndecan-1 inhibition of the MEK-Erk pathway. Carcinogenesis. 2011;32:1518-24.

89. Elledge RM, Green S, Pugh R, Allred DC, Clark GM, et al. Estrogen receptor (ER) and progesterone receptor $(\mathrm{PgR})$, by ligand-binding assay compared with $\mathrm{ER}, \mathrm{PgR}$ and $\mathrm{pS} 2$, by immuno-histochemistry in predicting response to tamoxifen in metastatic breast cancer: a Southwest Oncology Group Study. Int J Cancer. 2000;89:111-7.

90. Abate-Shen C, Shen MM. Molecular genetics of prostate cancer. Genes Dev. 2000;14:2410-34.

91. Shapiro CL, Manola J, Leboff M. Ovarian failure after adjuvant chemotherapy is associated with rapid bone loss in women with early-stage breast cancer. J Clin Oncol. 2001;19:3306-11.

92. Maillefert JF, Sibilia J, Michel F, Saussine C, Javier RM, et al. Bone mineral density in men treated with synthetic gonadotropinreleasing hormone agonists for prostatic carcinoma. J Urol. 1999;161:1219-22.

93. Hubner A, Mulholland DJ, Standen CL, Karasarides M, Cavanagh-Kyros J, et al. JNK and PTEN cooperatively control the development of invasive adenocarcinoma of the prostate. Proc Natl Acad Sci U S A. 2012;109:12046-51.

94. Chen CD, Welsbie DS, Tran C, Baek SH, Chen R, et al. Molecular determinants of resistance to antiandrogen therapy. Nat Med. 2004;10:33-9.

95. McEntee MF, Ziegler C, Reel D, Tomer K, Shoieb A, et al. Dietary n-3 polyunsaturated fatty acids enhance hormone ablation therapy in androgen-dependent prostate cancer. Am J Pathol. 2008;173: $229-41$.

96. Friedrichs W, Ruparel SB, Marciniak RA, deGraffenried L. Omega-3 fatty acid inhibition of prostate cancer progression to hormone independence is associated with suppression of mTOR signaling and androgen receptor expression. Nutr Cancer. 2011;63:771-7.

97. Cooke M, Di Consoli H, Maloberti P, Cornejo Maciel F. Expression and function of OXE receptor, an eicosanoid receptor, in steroidogenic cells. Mol Cell Endocrinol. 2013;371:71-8.

98. Marjon NA, Hu C, Hathaway HJ, Prossnitz ER. G proteincoupled estrogen receptor regulates mammary tumorigenesis and metastasis. Mol Cancer Res. 2014;12:1644-54.

99. Cao W, Ma Z, Rasenick MM, Yeh S, Yu J. N-3 poly-unsaturated fatty acids shift estrogen signaling to inhibit human breast cancer cell growth. PLoS One. 2012; 7:e52838.

100. Manni A, Xu H, Washington S, Aliaga C, Cooper T, et al. The impact of fish oil on the chemopreventive efficacy of tamoxifen against development of N-methyl-N-nitrosourea-induced rat mammary carcinogenesis. Cancer Prev Res (Phila). 2010;3:32230.

101. Sverrisdottir A, Fornander T, Jacobsson H, von Schoultz E, Rutqvist LE. Bone mineral density among premenopausal women with early breast cancer in a randomized trial of adjuvant endocrine therapy. J Clin Oncol. 2004;22:3694-9.

102. Eriksson S, Eriksson A, Stege R, Carlstrom K. Bone mineral density in patients with prostatic cancer treated with orchidectomy and with estrogens. Calcif Tissue Int. 1995;57:97-9.

103. Orchard TS, Ing SW, Lu B, Belury MA, Johnson K, et al. The association of red blood cell n-3 and n-6 fatty acids with bone 
mineral density and hip fracture risk in the women's health initiative. J Bone Miner Res. 2013;28:505-15.

104. Moon HJ, Kim TH, Byun DW, Park Y. Positive correlation between erythrocyte levels of n-3 polyunsaturated fatty acids and bone mass in postmenopausal Korean women with osteoporosis. Ann Nutr Metab. 2012;60:146-53.

105. Banu J, Bhattacharya A, Rahman M, Kang JX, Fernandes G. Endogenously produced n-3 fatty acids protect against ovariectomy induced bone loss in fat-1 transgenic mice. J Bone Miner Metab. 2010;28:617-26.

106. Chen TY, Zhang ZM, Zheng XC, Wang L, Huang MJ, et al. Endogenous n-3 polyunsaturated fatty acids (PUFAs) mitigate ovariectomy-induced bone loss by attenuating bone marrow adipogenesis in FAT1 transgenic mice. Drug Des Dev Ther. 2013;7: 545-52.

107. Rahman MM, Bhattacharya A, Banu J, Kang JX, Fernandes G. Endogenous n-3 fatty acids protect ovariectomy induced bone loss by attenuating osteoclastogenesis. J Cell Mol Med. 2009;13: 1833-44.

108. Orchard TS, Pan X, Cheek F, Ing SW, Jackson RD. A systematic review of omega-3 fatty acids and osteoporosis. Br J Nutr. 2012;107 Suppl 2:S253-60.

109. Steffens JP, Herrera BS, Coimbra LS, Stephens DN, Rossa Jr C, et al. Testosterone regulates bone response to inflammation. Horm Metab Res. 2014;46:193-200.

110. Gao L, Faibish D, Fredman G, Herrera BS, Chiang N, et al. Resolvin E1 and chemokine-like receptor 1 mediate bone preservation. J Immunol. 2013;190:689-94.

111. Hofbauer LC, Schoppet M. Clinical implications of the osteoprotegerin/RANKL/RANK system for bone and vascular diseases. JAMA. 2004;292:490-5.

112. Gullett NP, Mazurak VC, Hebbar G, Ziegler TR. Nutritional interventions for cancer-induced cachexia. Curr Probl Cancer. 2011;35:58-90.

113. Barber MD, Ross JA, Voss AC, Tisdale MJ, Fearon KC. The effect of an oral nutritional supplement enriched with fish oil on weightloss in patients with pancreatic cancer. Br J Cancer. 1999;81:80-6.
114. Colomer R, Moreno-Nogueira JM, Garcia-Luna PP, Garcia-Peris $\mathrm{P}$, Garcia-de-Lorenzo A, et al. N-3 fatty acids, cancer and cachexia: a systematic review of the literature. Br J Nutr. 2007;97:82331.

115. Fearon KC, Von Meyenfeldt MF, Moses AG, Van Geenen R, Roy A, et al. Effect of a protein and energy dense N-3 fatty acid enriched oral supplement on loss of weight and lean tissue in cancer cachexia: a randomised double blind trial. Gut. 2003;52: 1479-86.

116. Fearon KC, Barber MD, Moses AG, Ahmedzai SH, Taylor GS, et al. Double-blind, placebo-controlled, randomized study of eicosapentaenoic acid diester in patients with cancer cachexia. $\mathbf{J}$ Clin Oncol. 2006;24:3401-7.

117. Jatoi A, Rowland K, Loprinzi CL, Sloan JA, Dakhil SR, et al. An eicosapentaenoic acid supplement versus Megestrol acetate versus both for patients with cancer-associated wasting: a North Central Cancer Treatment Group and National Cancer Institute of Canada collaborative effort. J Clin Oncol. 2004;22:2469-76.

118. Mantovani G. Randomised phase III clinical trial of 5 different arms of treatment on 332 patients with cancer cachexia. Eur Rev Med Pharmacol Sci. 2010;14:292-301.

119. Sorensen LS, Thorlacius-Ussing O, Rasmussen HH, LundbyeChristensen S, Calder PC, et al. Effects of perioperative supplementation with omega-3 fatty acids on leukotriene $\mathrm{B}(4)$ and leukotriene $\mathrm{B}(5)$ production by stimulated neutrophils in patients with colorectal cancer: a randomized, placebo-controlled intervention trial. Nutrients. 2014;6:4043-57.

120. Bougnoux P, Hajjaji N, Ferrasson MN, Giraudeau B, Couet C, et al. Improving outcome of chemotherapy of metastatic breast cancer by docosahexaenoic acid: a phase II trial. Br J Cancer. 2009;101:1978-85.

121. Aronson WJ, Kobayashi N, Barnard RJ, Henning S, Huang $\mathrm{M}$, et al. Phase II prospective randomized trial of a low-fat diet with fish oil supplementation in men undergoing radical prostatectomy. Cancer Prev Res (Phila). 2011;4:206271. 